\title{
Pharmacy Assistant Mobile Application (PAMA): Development and Reviews
}

\author{
https://doi.org/10.3991/ijim.v11i3.6757 \\ Orawit Thinnukool \\ Chiang Mai University, Chiang Mai, Thailand \\ orawit.t@cmu.ac.th \\ Pattaraporn Khuwuthyakorn \\ Chiang Mai University, Chiang Mai, Thailand \\ pattaraporn.khuwuth@cmu.ac.th \\ Purida Wientong \\ Chiang Mai University, Chiang Mai, Thailand \\ purida.v@emu.ac.th
}

\begin{abstract}
Nowadays, pharmaceutical mobile applications are widely used. Several features and functionality play an important role to support the real needs of users especially in primary medication. Users' behaviors in the modern world have changed where users may prefer to access drug information using search engines via the Internet rather than consulting with professionals like pharmacists, doctors or experts. However, the drug information that users retrieve from the internet sources may provide inaccurate, incomplete or unreliable information.

The questions are: can we decrease this phenomenon? Suppose that we are applying an application to a content provider, which application functionalities are suitable for users and support their real needs? Can the application encourage users to gather drug information via the application instead of searching via the internet sites? The proposed study aimed to develop a Pharmacy Assistant Mobile Application (PAMA) based on necessarily required features and functionalities which are designed and operate on the iOS operation system. The application performance has been tested and measured regarding the graphic user interface and the system acceptance level.

The experimental results have been reviewed and an issue has been found which needs to be considered as an important factor when developing a healthcare mobile application for the real uses.
\end{abstract}

Keywords—application performance, mobile application, pharmacy, assistant

\section{$1 \quad$ Introduction}

Modern technology nowadays, especially smartphone technology, has several advantageous functions, faster computational time, wider screens, higher capability, 
etc[1][2]. The estimation of the smartphone usage is about two billion devices which are utilized in everyday life, and it has been predicted in 2018, the number of smartphone users worldwide will increase to six billion people [3][4][5].

Mobile technology has played a huge role for users which includes, for example, offering them the information to track their health conditions, providing a suggestion on healthcare medication and being tools for medical providers for monitoring their patients. Several benefits from healthcare functions on mobile apps have been worthwhile for the secondary treatment in hospital or clinic. Additionally, smartphones are used for tracking or managing patients' health [1][2][6].

Although the exciting screen designs of mobile apps such as text fonts, colors and various animations which are displayed in the user interface attract users to download the application easier than other factors [7], the key point for user consideration in choosing a healthcare application is the design of features and functionalities. So, the challenging questions in design become: can functions support the real needs of users? Do they have clear understanding in each function of the application? In term of usability, can the healthcare application support them in self-medication etc. [8].

Nowadays, patients are less likely to get medical advice from doctors or medical experts [9][10]. Research indicates $19 \%$ of smartphone users have at least one healthcare application on their mobile devices, and more than $52 \%$ have used their devices for accessing medication information or searching for advice on healthy living via the Internet [11].

The report of Joseph and his colleagues indicates another concern of obtaining health information via the Internet, it states that $59 \%$ of users did not want to consult doctors [12]. While the Internet information trust leads to possible dangers of purchasing drug over the Internet which might occur to patients due to mistaken information, fake information, different active ingredients and dangerous ingredients etc. [13]. The recent report of Lombardo and Cosentino claims the Internet use for searching drug information is popular in Italy [14]. About $87.5 \%$ of 1,008 Italian participants have rated satisfactory retrieval of information over the Internet. Similar to the report of Donna and his colleagues, it shows users trust in the Internet as a source of health information for medication [15]. Note that the experiment did not consider online pharmacies which are legally operated by pharmacists or experts.

We cannot ignore this phenomenon since users can find drug information by themselves on the Internet sources. The report of Bill confirmed when consumers obtain drug information over the Internet, they tend to receive a lack of appropriate drug and medication information [16]. The questions are can we believe the information from websites?. Does the information work for primary medication? Although selfmedication could be beneficial for primary treatment, some information from search engines could be uncertain, as it contains incorrect and garbage information. If patients follow the false information, it could cause them serious danger. The mobile healthcare application could be a trustworthy content provider for self-medication, but we need to promote and encourage users to use the healthcare application instead of searching high risk information over the Internet sites.

The application of mobile pharmacy application has provided greatly benefits to patients with reliable information for treatment which can be used for meditation prior 
to the secondary treatment at hospitals or clinics [17][18]. Moreover, it can suggest drug information and various pharmaceutical products [8][19][20][21]. However, the challenging design of features and functionalities of the mobile healthcare application are the key factor which could indicate the success of the development. How to deliver useful functions to support users for their life? How to encourage users to apply the healthcare mobile application as a tool for providing drug information instead of using search engines over the internet site.

Additionally, the level of acceptance, attitude of users when using the application or encountering to the new technology while promoting and their understanding need to be considered. Thus, for the new development, we carefully considered what features and functionalities are needed to service users and also to decrease the webbased searching behavior for drug information.

In this paper, the study aimed to (1) develop the Pharmacy Assistant Mobile Application (PAMA) for primary medication and (2) measure the application performance in term of satisfactory acceptance and users' attitude towards the developed features and functionalities.

\section{Material and methods}

\subsection{Medicine Contents}

The PAMA database includes over-the-counter (OTC) products commonly sold in Chiang Mai university drugstores which are household medicines, topical nondangerous drugs, dietary supplements herbs and alternative medicines. The data covers 226 products' information designed to provide important aspects of data from medical literature and manufacturers in concise format. The products are classified by laws and by the way they are used to treat a particular condition. The list of product categories is shown in Table 1.

Table 1. List of product categories.

\begin{tabular}{|l|l|}
\hline \multicolumn{2}{|c|}{ Product categories } \\
\hline Topical medicine & Household medicines \\
Wound healing medicine & Non-dangerous drugs \\
Antiseptics & Vitamins \\
Abdominal pain & Dietary supplements \\
Laxative & Cosmetics \\
\hline
\end{tabular}

\subsection{Research Operation}

Firstly, the experiment was conducted by developing a questionnaire for interviewing users about their opinions and attitudes towards using mobile healthcare application in primary medication. Table 3 demonstrates general questions of the questionnaire which aim to understand users' background in drug usage behaviors in everyday life. The next section of the questionnaire, in Table 4, asks for users' attitudes towards 
the use of mobile applications as a tool in the primary healthcare treatment. After the PAMA was fully developed it was released for users in Chiang Mai University drug store.

Table 5 shows questions asked of respondents to try the application by providing them testing devices. This section aims to understand users' satisfaction of the PAMA in term of UI design and its performance when applied to their healthcare medication. Finally, the last section of the questionnaire in Table 6 is for testing the user acceptance of the PAMA in healthcare medication. It targets to encourage users to use the PAMA instead of searching drug information from the Internet.

The experiment has been conducted using two groups of pharmacy customers with a total of 61 people as the samples. The first group is comprised of 31 customers who purchase medicines from the drugstore of Chiang Mai University. The remaining samples are the second group which is the customers of other drugstores within Chiang Mai province. The PAMA has been released on Apple's App Store for testing and their opinions and attitudes over the PAMA has been collected.

\subsection{Application Development}

The PAMA has been developed adopting the adapted waterfall methodology. One of the application functions is the barcode scanner. We applied the barcode scanner together with the Internet connection as a technique to enable the PAMA retrieving ability of drug information from the barcodes appeared on drug boxes. Moreover, the PAMA provides other useful functions to support users such as the function that users can retrieve all information about drugs used for their medication when the drug information is missing and the alarm function to remind users when medication needs to be taken, etc. The list of functionalities of the PAMA is shown in Table 2.

Table 2. Functionalities of the PAMA

\begin{tabular}{|l|l|l|}
\hline \multicolumn{1}{|c|}{ Function } & \multicolumn{1}{c|}{ Propose } & \multicolumn{1}{c|}{ How to use } \\
\hline $\begin{array}{l}\text { Scan } \\
\text { (Fig. 2.) }\end{array}$ & $\begin{array}{l}\text { Display drugs' information } \\
\text { such as using information/ } \\
\text { pricing / pharmacy name }\end{array}$ & $\begin{array}{l}\text { The user has to go to scanning function and } \\
\text { scan barcode from drugs (box), the application } \\
\text { will display drugs information/ use can make } \\
\text { drug information to favorite for taking alarm. }\end{array}$ \\
\hline $\begin{array}{l}\text { (b) Alarm } \\
\text { (Fig. 3.) }\end{array}$ & $\begin{array}{l}\text { Scan set alarm function for } \\
\text { notification to take medicine. }\end{array}$ & $\begin{array}{l}\text { Setting time for warning when it is time to take } \\
\text { medicine time. }\end{array}$ \\
\hline $\begin{array}{l}\text { (c) Search } \\
\text { (Fig. 2.) }\end{array}$ & $\begin{array}{l}\text { Search a name of drug infor- } \\
\text { mation by typing by keyword }\end{array}$ & $\begin{array}{l}\text { User goes to search function, and types key- } \\
\text { word into textbox for searching a drug infor- } \\
\text { mation }\end{array}$ \\
\hline $\begin{array}{l}\text { (d) Note reminder (Memo) } \\
\text { (Fig. 3.) }\end{array}$ & Recording any information & $\begin{array}{l}\text { Typing any notes and saving or deleting any } \\
\text { information }\end{array}$ \\
\hline
\end{tabular}

Fig.1 shows screenshots of the PAMA, where the left panel is the main menu which contains four functions. The middle panel shows the user guide and warning of the PAMA. The right panel shows the barcode scanner function for searching drug information online which supports users for finding correct information more conveniently than getting access to the information via the Internet. 

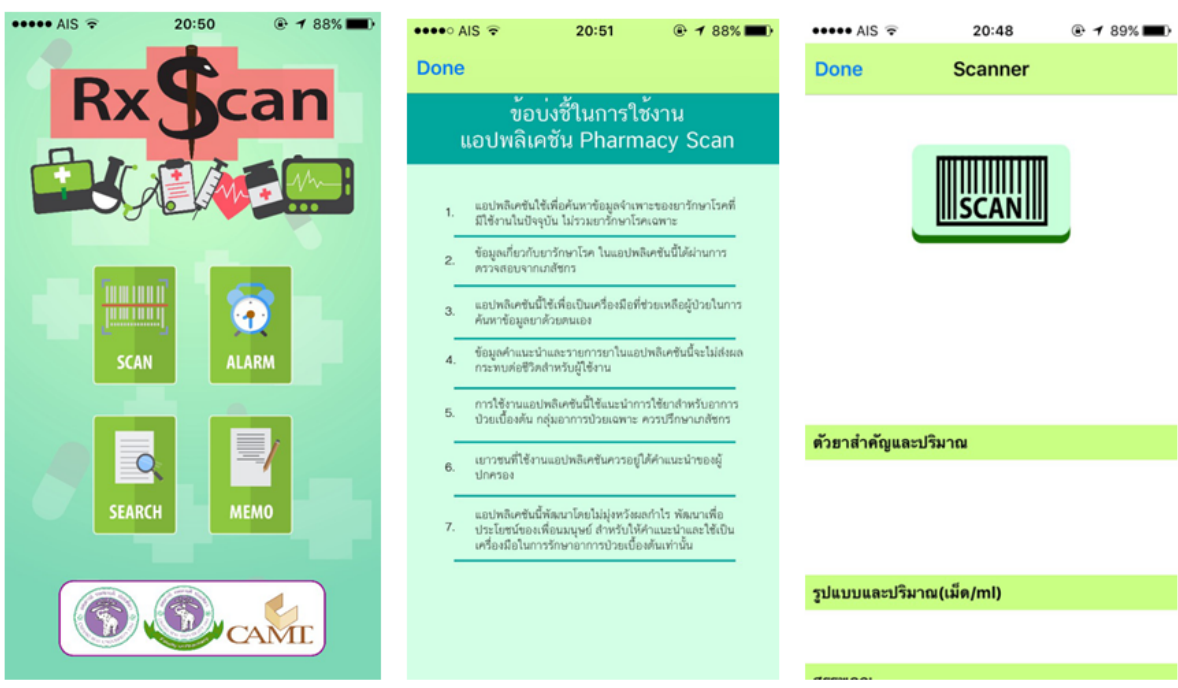

Fig. 1. Screenshots of the PAMA, from left to right, are the main menu, user guide and the barcode scanner function.

In Fig. 2, screenshots include (on the left) the barcode scanner function when scanning over a barcode on a drug box, (in the middle) the result of the barcode scanner searching which shows the drug information corresponding to the scanned medication barcode and (on the right) the search function using keywords which allows users to find detailed drug information by drug name, drug type or symptoms.
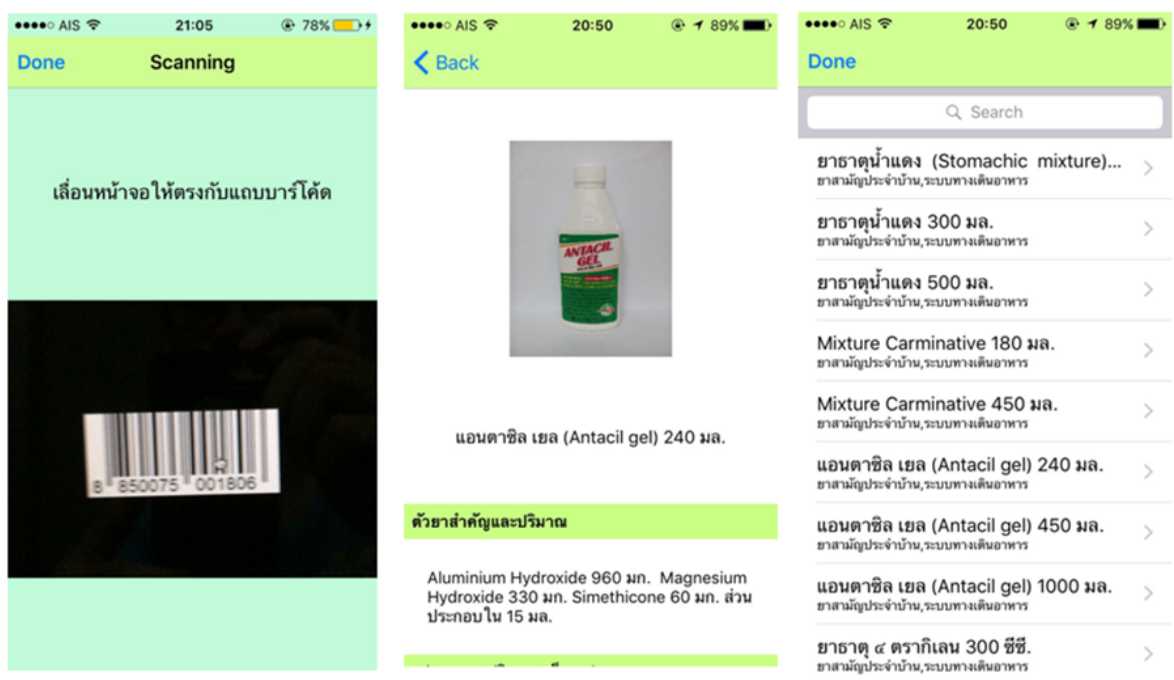

Fig. 2. Screenshots show the barcode scanning function, the result and the searching function using keywords. 

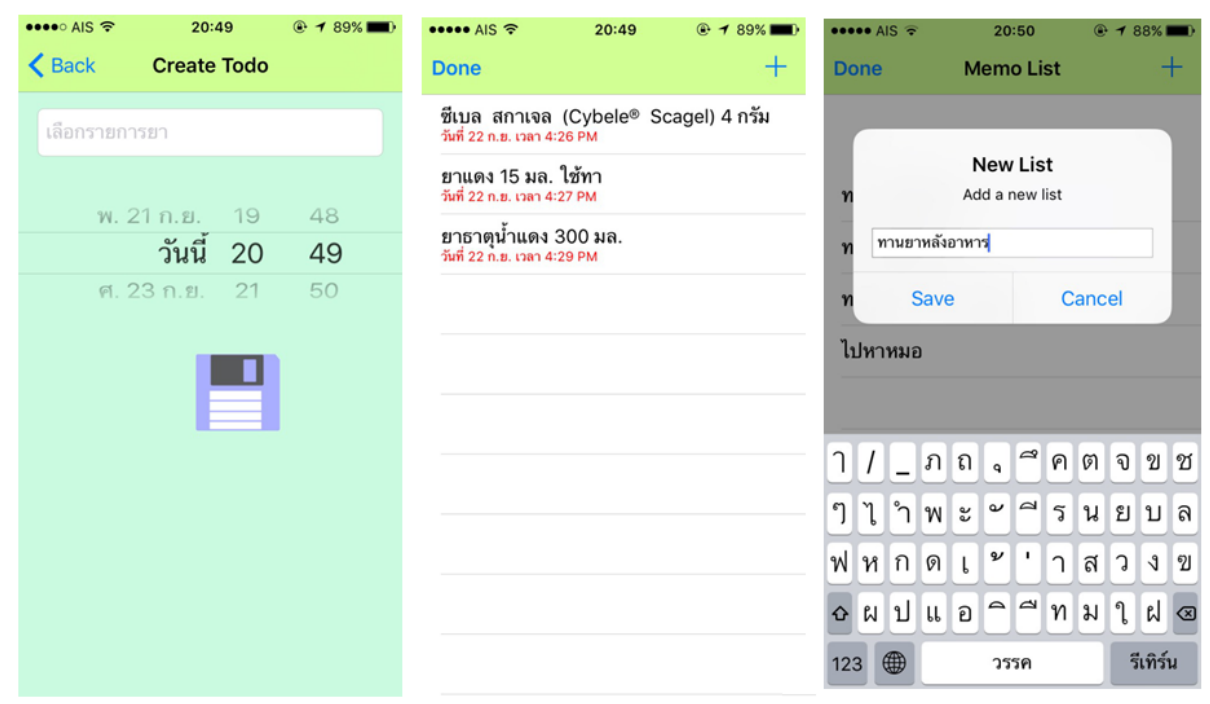

Fig. 3. Screenshots show the alarm setting function and the memo function.

Fig. 3 indicates the alarm setting function. On the left panel, it begins with searching a drug name, then the detail will appear as in the middle panel. After that, users can set up the time for the reminders or alarms. On the right panel, it shows the memo function which users can use for written notes or to record any medication information.

\section{$2.4 \quad$ Application Reviews}

After each function of the PAMA, it was reviewed and tested by 11 pharmacists who are specialists from the Faculty of Pharmacy, Chiang Mai University. The comments are as follows;

Comment group 1: Users have concerns about the provided drug details, where some of the information still needs to be advised by pharmacists or under guidance of experts.

Comment group 2: They have concerns about the attitude of users, when the information suggested from the PAMA is different from pharmacists.

Comment group 3: They have concerns about which target group the PAMA was developed for.

Comment group 4: The number of drug information is limited.

Comment group 5: User Interface (UI) Suggestion includes the size of text and pictures should be increased.

Comment group 6: The drug information of the PAMA could be more detailed than what is present on the drug boxes.

Table 3 to Table 6 are questions that follow criteria for interviewing and evaluating the graphic user interface (GUI), system performance and system confidence. 
Paper-Pharmacy Assistant Mobile Application (PAMA): Development and Reviews

Table 3. List of questions to ask respondents for general information.

\begin{tabular}{|l|}
\hline \multicolumn{1}{|c|}{ Questions } \\
\hline G1- Sex \\
\hline G2- Type of sample group [student / general group] \\
\hline G3- Age 15-20 [ ]21-25 [ ]26-30 [ ]31-35 [ ]36-40 [ ]41-45 [ ] 45-50 [ ] 50 up \\
\hline G4- Residency [within Chiang Mai Municipality areas / Outlying districts] \\
\hline $\begin{array}{l}\text { G5-Education [lower than bachelor's degree/bachelor's degree/higher than bachelor's } \\
\text { degree] }\end{array}$ \\
\hline G6:1-Mobile device usage Android [ ] iOS [ ] Windows [ ] \\
\hline G6:2-Your smart phone provides assistance in helping you to find information. \\
\hline G6:3-You have ever known an application that provides health information \\
\hline G6:4-Your smart phone has an application relating to health information. \\
\hline G6:5-You paid for the health application \\
\hline G7:1-You have experience in self-medication. \\
\hline G7:2-You buy medicines from pharmacists at drug stores. \\
\hline G7:3-You searching from the internet or the application before buying or using drugs. \\
\hline G7:4-You choose to use self-medication without consulting doctors or pharmacist. \\
\hline G7:5-Consulting with pharmacist is not necessary for you to use drugs. \\
\hline G7:6- How often for searching drug information via internet \\
\hline $\begin{array}{l}\text { G7:7-The reasons that you use self-medication / (A: recurrent illnesses, B:minor symptom illnesses, C: } \\
\text { timesaving and D: money saving) }\end{array}$ \\
\hline
\end{tabular}

Table 4. List of questions to ask users about their attitude towards the use of mobile applications as a tool in the primary care treatment.

\footnotetext{
Questions

Q22:1- Using healthcare applications could be a tool in providing information on primary care treatment more than searching from internet site.

Q22:2-Using healthcare applications would provide positive primary care treatment results.

Q22:3-Using healthcare applications could provide precise information for healthcare treatment as same as the professional advice from doctors or pharmacists.

Q22:4-Using healthcare applications could help making better self-healthcare.

Q22:5-Using healthcare applications would change behavior and motivate users in self-healthcare practice. Q22:6-Using healthcare applications would not cause serious consequences to the health of users.

Q22:7-Using healthcare applications would help users in reducing the healthcare costs when illness occurs. Q22:8- Using healthcare applications would facilitate users in primary treatment.
}

Table 5. List of questions to ask respondents after letting them try the PAMA by providing testing devices. (5-10 minutes, then allow the users to answer questions)

\section{UT Evaluation}

\section{Questions}

Q3:1- Press buttons on the screen are easy to use.

Q3:2- Screen size clearly shows foreground and background

Q3:3- The screen colors are suitable

Q3:4- Each image of the menu communicates with users effectively 
Q3:5- The number of the menu items is suitable and easy to use

Q3:6- The font size is suitable for the screen and easy to read

Q3:7- Textured wallpapers are suitable

Q3:8- The proportion of display areas is appropriate

Q3:9- The contrast of the display screen is beautiful

Q3:10-The alignments of graphics and symbols are easy to understand

\section{System Performances}

Q4:1- The application is intuitive and understandable for users on the trial

Q4:2- The application provides useful information in medication for your primary self-treatment

Q4:3- The barcode scanner function could help you to find medicines easier

Q4:4- The medication notification function is useful for treatment

Q4:5- Search function using keywords provides easier searching tool

Q4:6- The function for recording how to take medicines (typing) help users to keep the records easier

Q4:7- Short note function helps you in recording related information in treatment easier

Q4:8- Information from the application enables users for self-medication

Q4:9-The application makes self-medication easier for users even without professional advices from

pharmacists.

Q4:10-The application can operate quickly on the smartphone and the system is stable (not stuck).

Table 6. List of questions to evaluate users' confidence in the PAMA functionalities.

Questions
Q5:1-User has confidence that the application works in providing advices for primary treatment
Q5:2-User has confidence that the medicines information in the application is precise
Q5:3-User has confidence that the application can provide precise information as same as the professional
advices such as pharmacists
Q5:4-User has confidence that the information from the application can help users to understand how to
use medicines and apply the information for the immediate treatment
Q5:5-User has confidence that the advices from the application will do no harm to users
Q5:6-You agree to use the application and will recommend it to friends as well
Q5:7- If you are in an area where there is no pharmacist, you will use the application for self-medication
Q5:8-You believe that the application can reduce healthcare costs when illness occurs
Q5:9- You believe that the application will facilitate users in primary healthcare treatments more than
searching information via the internet.
Q5:10-User has confidence to download the application or recommend it to other

\section{Results}

The PAMA has been reviewed in the research experiment by using the questionnaire. Then, the application has been suggested to users, while they were buying drugs in Pharmacy shops. Fig. 4 shows the use of the PAMA in a Pharmacy shop for searching drug information. The PAMA is recommended to download from the App Store. 

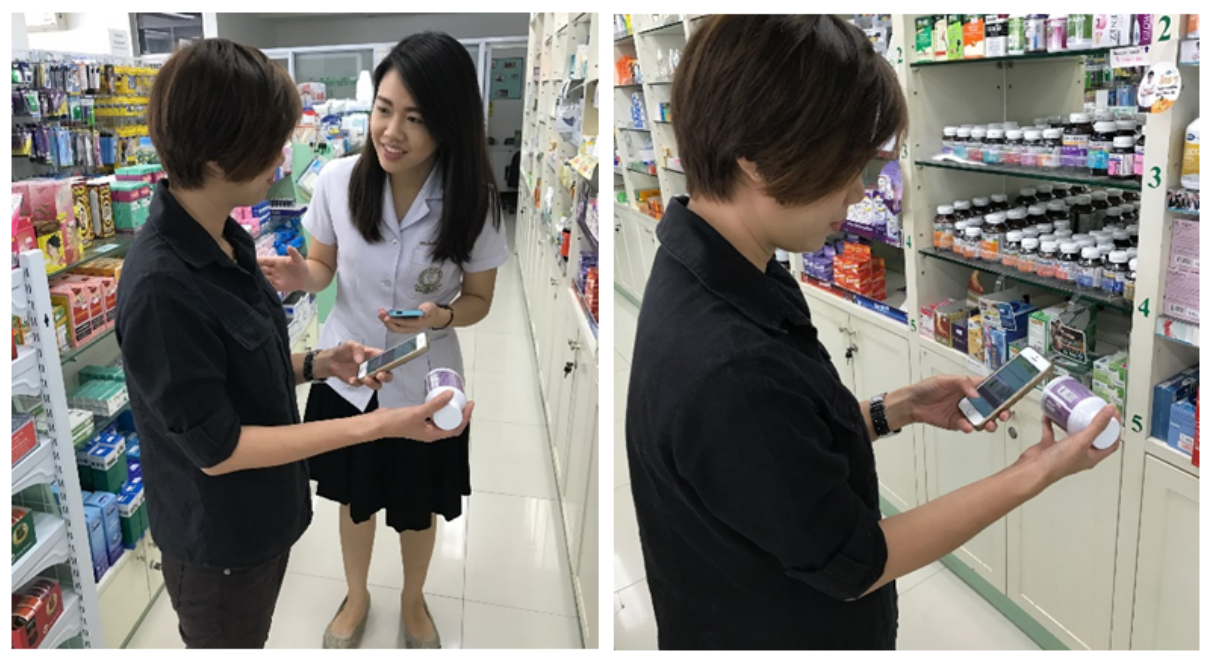

Fig. 4. A user using the PAMA

The experimental result is in Fig. 5. The upper panel indicates the results in the section one of the questionnaire which includes general questions. The majority of the respondents are female $(78.8 \%)$. While $75.8 \%$ of respondents are general people and over one-fifth $(24.2 \%)$ are students. The largest proportion $(16.7 \%)$ of respondents are between 21-25 years old, the second largest are between 31 to 35 (14.3\%). In regard to residency areas, the majority of respondents $(51.5 \%)$ are living in the outlying municipality area. More than half $(54.5 \%)$ of respondents graduated with a bachelor degree, while the rest of respondents have lower levels of education.

The lower panel of Fig. 5 presents the information of mobile device usage and the users' experiences in healthcare applications. From Left to Right is the result of questions aimed to ask respondents about their knowledge in healthcare applications. It shows the majority of respondents $(57.1 \%)$ use Android Operating System. The next one shows $84.8 \%$ of respondents agree to use the mobile phones via web browsers for searching any information online. Additionally, $48.5 \%$ of respondents have known or experienced at least one of the healthcare applications, while $51.5 \%$ of them have never had any chance to see one. More than half (57.6\%) have installed at least one healthcare application on their mobile devices. When asking for more details about the downloaded healthcare application, $78.6 \%$ did not purchase the application. The final question was about users' experience of buying drugs by themselves. The result shows the majority of the respondents $(78.6 \%)$ still consult with pharmacists or experts for medication. 

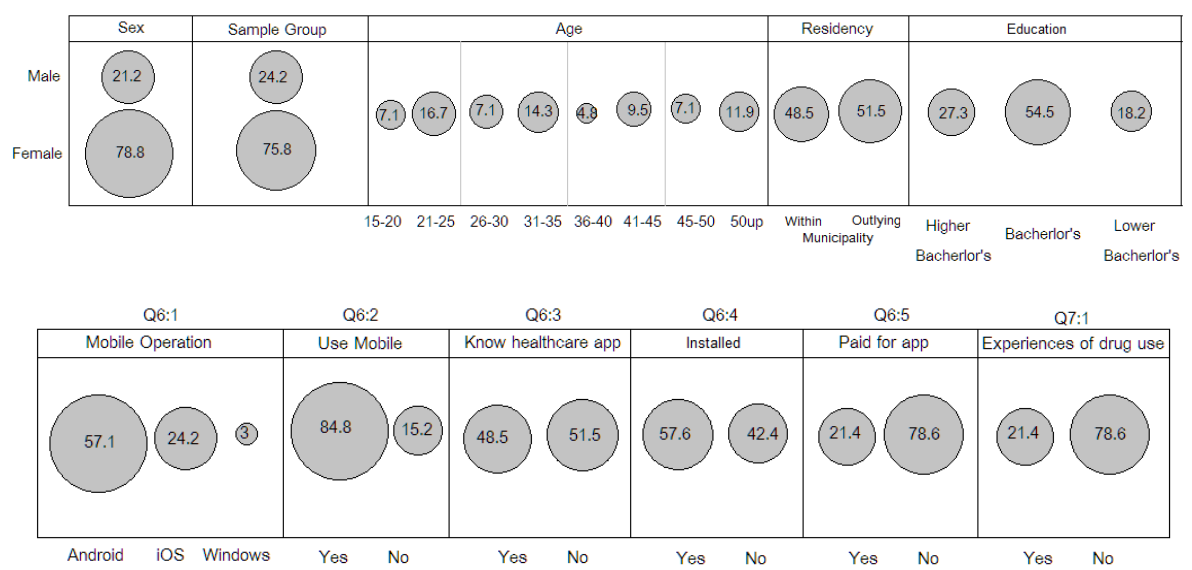

Fig. 5. The questionnaire result shown in percentage of responses: the top row is the general information of respondents and the bottom row is the information of mobile device usage and the users' experiences in healthcare applications.

Fig. 6 shows the information of self-initial medication behavior. Q7:2 shows up to $21.4 \%$ of respondents always do self-medication. While the highest proportion of $28.6 \%$ do self-medication very often and $21.4 \%$ do it sometimes. When asking respondents whether they search for drug information over the Internet or application before buying medicines in Q7:3, the result show $26.2 \%$ do searching sometimes before buying and $19.0 \%$ do searching very often to buy medicines. When asking users how often they use self-medication $\mathrm{Q} 7: 4$, it has been found that $23.8 \%$ of respondents sometime use self-medication without consulting doctors or pharmacists similar to the number of respondents who rarely use self-medication. Next question, Q7:5, it shows $31 \%$ of respondents agree to consult with pharmacists, whereas $26.2 \%$ of them consult pharmacists sometimes. When asking respondents about how often they do searching for drug information over the Internet, $28.6 \%$ said they search over the Internet very often, while others (28.6\%) have never tried it before (Q7:6). On the right column of Fig.6, Q7:7 is a multiple-answer question which shows users' reasons of using self-medication. The majority of $72.7 \%$ of respondents use self-medication for recurrent illnesses, while $57.1 \%$ believe that they could save time for medication if they use self-medication.

Fig. 7 shows the users' attitudes towards the use of mobile applications as a tool for primary care treatment after the collection of the responses using the Likert Scale. Questions in this section focus on general mobile healthcare applications. Regarding the results of Q22:1 to Q22:8, the majority of respondents agreed in all questions that mobile applications could be an appropriate tool in the primary care treatment. In Q22:8, more than $21.27 \%$ agreed or strongly agreed that mobile healthcare application could also facilitate users in primary treatment. When considering Q22:3, it shows $42.4 \%$ moderately agreed, but $30.3 \%$ disagreed that using healthcare applications could provide precise information as same as the advice from professionals, doctors or pharmacists. In Q22:4, 42.4\% moderately agreed that using mobile 
healthcare applications could help make better self-healthcare, whereas $36.6 \%$ disagreed.

The interviews have been conducted after letting correspondents test the PAMA. Fig. 8 shows the interview results which are from section 3 of the questionnaire. The questions in this section reviewed the PAMA user interface (UI). Results of overall user agreement show the majority of responders had the satisfactory results. However, the point of consideration is in Q3:6 to Q3:8 which show the results of more than 30\% of users who answered the moderate level of agreement rather than "agree" or "strongly agree". The issue can be improved in the next development to increase the level of agreement in UI design.

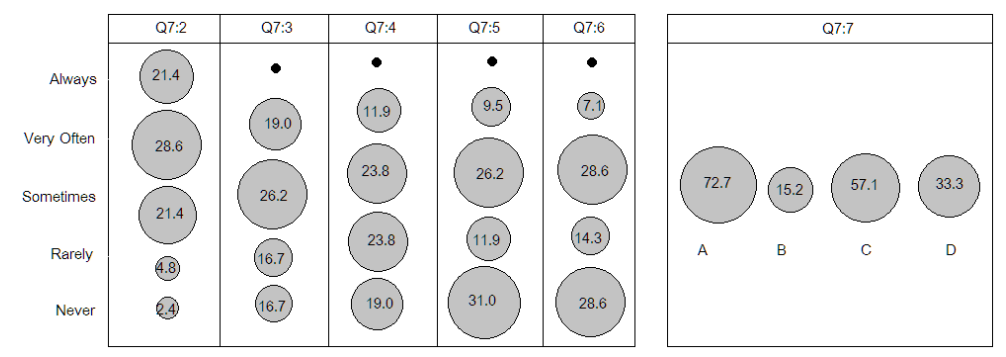

Fig. 6. The distributions of the percentage of level agreements corresponding to the questionnaire section of self-initial medication behavior (Table 3 ).

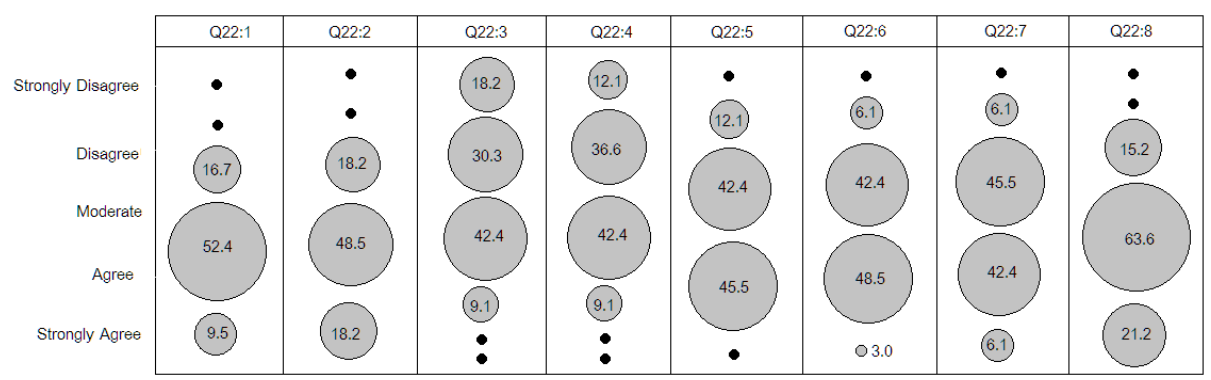

Fig. 7. The distributions of the percentage of level agreements corresponding to users' attitude towards the use of mobile applications as a tool in the primary care treatment (Table 4).

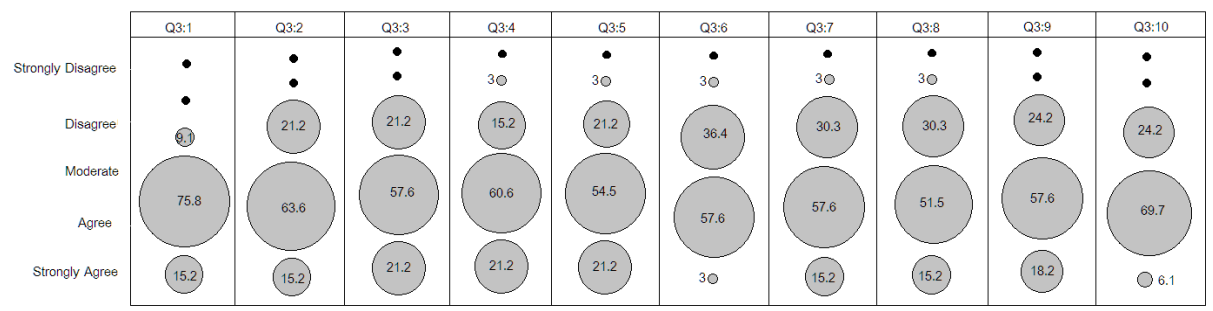

Fig. 8. The distributions of the percentage of agreement corresponding to the UI feedback from respondents after trying the PAMA by providing testing devices (Table 5). 
The result in Fig. 9 shows the percentages of agreement corresponding to the system performance of the PAMA. In Q4:1, the majority of users, $57.6 \%$ and $24.2 \%$, agreed, and strongly agreed, respectively, that the PAMA is intuitive and understandable. Whereas, Q4:3 indicates $24.2 \%$ of users strongly agreed that the barcode scanner function is useful to find medicines. According to Q4:4 to Q4:6, the results show $12.1 \%, 15.2 \%$ and $9.1 \%$ strongly agreed with the system performance of the notification function, searching function and recording function, respectively. The majority of respondents, $15.2 \%$ and $45.5 \%$, agreed and strongly agreed in Q4:7 which asks about the system performance for the short note function. In Q4:8, the result shows $63.6 \%$ of respondents agreed and strongly agreed that the information provided by the PAMA can enable users for self-medication as well as in Q4:9, the result shows that more than half of respondents agree and strongly agreed that using the PAMA could make self-medication easier for users, even there is no face-to-face professional advice. Moreover, respectively $39.4 \%$ and $18.2 \%$ of respondents agreed and strongly agreed that the PAMA can operate quickly and reliably on smartphones.

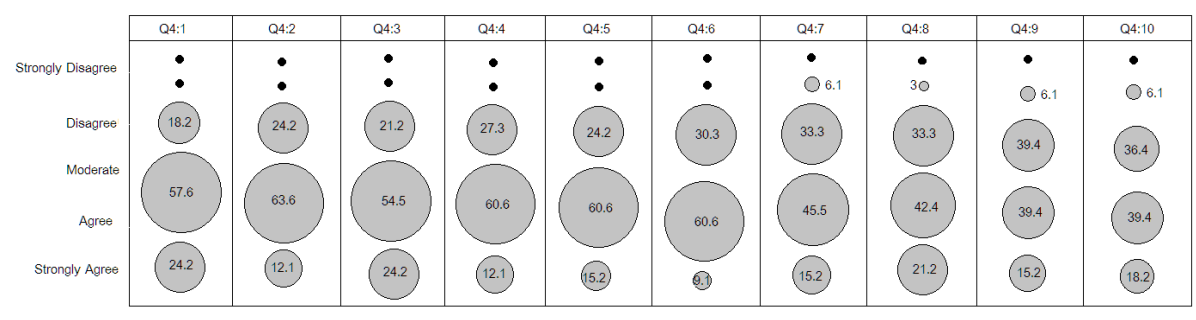

Fig. 9. The distributions of the percentage of agreement corresponding to the feedback of the system performance of the PAMA by providing testing devices (Table 5).

The results shown in Fig. 10 demonstrate the distribution of agreement corresponding to users' confidence in the PAMA after a trial. It shows users' confidence in Q5:1 to Q5:2 were satisfied with confidence with using the PAMA for healthcare medication. When asking whether the PAMA could provide precise information as same as professional advices or not (Q5:3), the majority of users agree and strongly agreed. Similar to Q5:4, when asking whether the information from the PAMA can help users for a better understanding of how to use medicines, more than $70 \%$ of users agree and strongly agreed. Moreover, in Q5:5, up to 78.8\% (include strongly agree and agree level) of users trusted that advice provided by the PAMA will do no harm to users, where over half of users answered in Q5:6 and Q5:7 that they will recommend the PAMA to their friends and are likely to use the application for self-medication when they are in an area without professional advice. Question Q5:8 asks whether users believe that the PAMA could reduce healthcare costs whether illness occurs or not. The result shows $63.5 \%$ of users strongly agreed. When asking users, in Q5:9, whether they believe that the PAMA could facilitate users in primary healthcare treatments better than the websites, $74.5 \%$ of respondents strongly agreed. In Q5:10, the result show that most users $(72.1 \%)$ would recommend the PAMA to other users. 


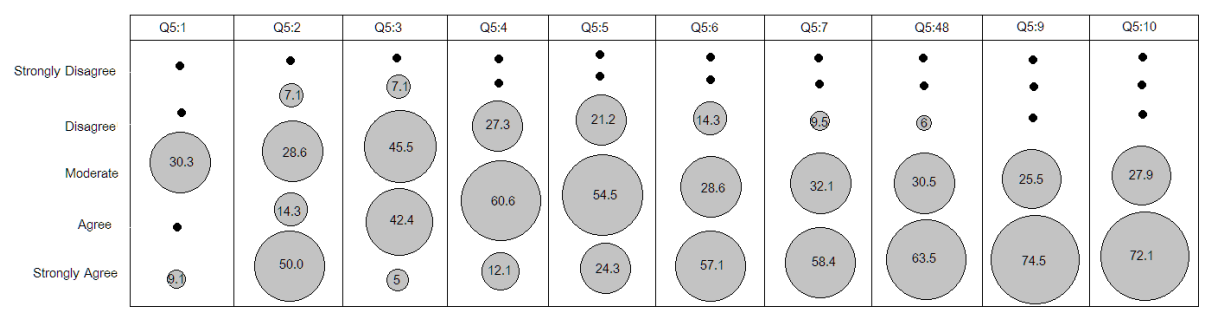

Fig. 10. The distributions of the percentage of agreement corresponding to users' confidence to the application functionalities (Table 6).

\section{Discussion}

The PAMA has been developed under the expectation of decreasing the use of search engines over the Internet for drug information. We considered the real needs of users when designing its functionalities which are necessary to support selfmedication and healthcare treatments and may decrease users' behavior in searching drug information over the Internet. The functionalities were concerned with corresponding to the challenge of appropriate features for daily life activities of users following the suggestion of Mariza and his colleagues [8]. Functions of the PAMA are mainly for searching drug instructions or drug information. Key word or habit search function enables easier access to drug information for users, then the list of the searching drug is displayed for providing the information to users. The drug information in the application contains the drug name, terms of use, preservation, indication, instruction, standard price (Prices are not available for all drugs). Moreover, there is the barcode scanner function to provide correct information to users, in the case of imported drug which may be difficult for Thai people to read or understand due to the language barrier. This function could serve users who need a good understanding of their drugs. The PAMA also includes other functions such as alarm and note reminder function which can support users to take drugs at a certain time of the day.

In the review section, the PAMA has been reviewed by pharmacists. Their comments are mostly concerned about whether the provided by PAMA should be used together with pharmacist or expert suggestion. Moreover, they concern about users' attitude towards the use of the PAMA. As it is not a pharmacist, most pharmacists disagreed about the use of the PAMA without professional advices, but the comments were different for non-dangerous drugs. Although the PAMA is able to function in providing adequate information about drugs, technology acceptance level among pharmacistsis still undesirable from the pharmacists' point of view. Not surprisingly, most mobile applications are not designed only for self-medication support, but are also an important likely to have optional tools to support interaction between pharmacists and patients. This issue is a good point for UX consideration. Moreover, the result shows that the PAMA users are satisfied in term of system performance, functionalities and user-interface which corresponds to the result of Alnanih, A. which 
claims the colorful design of features of applications is more likely to draw users' attention [7].

Although the PAMA could help users for primary medication, in Fig. 7 at Q22:3 and Q22:4, the users' attitudes show that they still needed professional advice along with the PAMA. In contrast, in Fig. 10, Q5:7 shows the majority of users (58.4\%) strongly agreed that they will use the PAMA for self-medication, if there is no pharmacist available in their areas. Moreover, users were confident to download the application and would recommend it to other people. Corresponding to Q5:3, 42.4\% of users had confidence that the PAMA could provide precise information, similar to professional advice. Both results indicate that the PAMA could be a choice for users to search for drug information instead of websites over the Internet.

Additionally, the positive attitude towards the healthcare application is able not only to change users' behavior in searching reliable drug information over the Internet, but also to promote users to use self-healthcare medication via the application. Although the decreases in users behavior in searching drug information over the Internet has not been evaluated yet, the result in Q5:9 indicates that users agreed that the precise information provided by the application can facilitate users in primary healthcare treatment. This implies that the PAMA could provide knowledge to users for warning of unreliable drug information from the Internet. Therefore, the PAMA has the potential to replace online search engines and websites and to decrease the use of unreliable drug information from the Internet.

Mobile healthcare applications have been rapidly growing. While many of them play an important role in medication to users nowadays, features and functions of application are still being developed to support users' needs different purposes. Although drug information used in everyday life has been increasingly gathered over the Internet [13][14], the PAMA development can also provide features and functions which are able to encourage users to use the application for searching instead of websites.

The problem of lack of appropriate drug information may be solved by the PAMA if users agree to use the application in their primary healthcare medication [16]. Correct information, useful content and interesting functions are key points to promote the PAMA. However, even if the PAMA is not applied to primary healthcare medication, it is still a tool to encourage users to pay attention their healthcare medication, make them concerned about using proper drugs and also motivate users to understand modern medication as well.

\subsection{Limitations}

Nonetheless, in the experiment, there were several constrains that should be improved as follows. First of all, the amount of background information of samples (respondents) was quite lacking because the sample size is small. The experiment should be improved by having a larger sample. Next, the period for application testing should be increased without our monitoring. Finally, the evaluation of the decreases of searching drug information over the Internet after using the PAMA was not con- 
ducted, and we did not consider online pharmacies or pharmacy websites which operate under pharmacists or experts.

\section{Conclusion}

The PAMA has been developed under the aim of developing the pharmacy assistant mobile application for primary medication. The challenging features and functionalities of the application were fully designed, implemented and has been released on the Apple's App Store. The application has evaluated system performance, in term of users' satisfaction. Then, the results show users' acceptance level and their attitudes of applying the PAMA with their primary medication.

In terms of using the PAMA instead of searching drug information over the Internet, the result indicates that users may understand and beware of incorrect information. However, the attitudes of experts and respondents regarding the use of the PAMA were different. In users' point of view, they agreed that there is value in using the PAMA for primary medication, while in the experts' point of view, they are concerned about applying the PAMA for the primary medication and they feel that the PAMA should be used under the advisory of pharmacists or experts.

\section{References}

[1] Emine, S. and Marco, S. (2009). Mobile health access for diabetics in rural Areas of Turkey - results of a survey.Lecture Notes of the Institute for Computer Sciences, Social Informatics and Telecommunications Engineering, 27:13-20

[2] Mohammad MosaA.S., Yoo, I. and Sheet, L. (2012). A systematic review of healthcare applications for smartphones.BMC Medical Informatics and Decision Making, 12(67): 131.

[3] Gertner. (2015). Smartphone users. ; June2, 2016 Available at: http://www.gartner.com /newsroom/id/2996817.

[4] Statista. (2015)a. Number of mobile phone users worldwide from 2012 to 2018 (in billions);May 31, 2015. Available at: http://www.statista.com/statistics/274774/for ecast-ofmobile-phone-users-worldwide.

[5] Statista. (2015)b. Number of smartphone users worldwide from 2012 to 2018 (in billions); May 31, 2015. Available at: http://www.statista.com/statistics/330695/number-ofsmartphone-users-worldwide.

[6] Raybardhan, S., Balen, R. M., Partovi, N., Loewen, P., Liu, G., and Jewesson, PJ. (2005). Documenting drug-related problems with PersonalDigital Assistants in a multisite health system. American Journal of Health-System Pharmacy, 62(17):1782-1787 https://doi.org/10.2146/ajhp040438

[7] Alnanih, A., Radhakrishnan, O., and Ormandjieva, O. (2012). Characterising context for mobile user interface in health care application. Procedia Computer Science, 10(2012): 1086-1093. https://doi.org/10.1016/j.procs.2012.06.153

[8] Mariza, M.B., Hamid, G., and Connolly, M. J. (2015). Mobile healthcare application: system design review, critical issues and challenges. Australasian Physical \&Engineering Sciences in Medicine, 38(1): 22-38. 
[9] Fox,BI.,Felkey, BG., Berger, BA., Krueger, KP., and Rainer, RK. (2007). Useof personal digital assistants for documentation of pharmacists'interventions: a literature review. American Journal of Health-System Pharmacy, 64(14):1516-1525 https://doi.org/10.2146/ ajhp060152

[10] Choi, A., Lovett, A.L.,Kang, J.,Lee, K., and Choi, L. (2015). Mobile application to improve medication adherence: existing apps, Quality of life and future direction. Advance in Pharmacology and Pharmacy, 3: 364-74 https://doi.org/10.13189/app.2015.030302

[11] Duggan, M., and Rainie, L. (2012). Cell phone activities 2012: additional demographic analysis. Pew Research Internet Project. November 25, 2012. Available at: http://www.pewinternet.org/2012/11/25/additional-demographic-analysis-2.

[12] Joseph, AD., Rebecca, AG., James, JN., Steven, ER., Peter, DF., and Anne. WM. (2002).

[13] Patients' Use of the Internet for Medical Information. Journal of General Internal Medicine, 17(3):180-185 https://doi.org/10.1046/j.1525-1497.2002.10603.x

[14] Cline, R. J. W. and Haynes K.M. (2001). Consumer health information seeking on the Internet: the state of the art. Health Education Research, 16(6): 671-692 https://doi.org/10.1093/her/16.6.671

[15] Lombardo, S. and Cosentino, M. (2016). Internet Use for Searching Information on Medicines and Disease: A Community Pharmacy-Based Survey Among Adult Pharmacy Customers.Interactive Journal of Medical Research, 13, 5(3):22

[16] Donna, MZ., Matthias, K., Zheng, K., and Lawrence, CA. (2011). Trust in the Internet as a Health Resource Among Older Adults: Analysis of Data from a Nationally Representative Survey. Journal of Medical Internet Research, 3(1):19

[17] Bill, K. (2015). Online pharmacies: buyer beware. Australian Prescriber, 38(6):186-187 https://doi.org/10.18773/austprescr.2015.067

[18] Pringle, J.L., Boyer, A., Conklin, M.H., McCullough, J.W., and Aldridge, A. (2014). The PennsylvaniaProject: pharmacist intervention improved medication adherenceand reduced healthcare costs. Health Affairs, 33(8):1444-1452 https://doi.org/10.1377/hlthaff.2013. 1398

[19] Tarek, M.E., Shazia, Q.J., and Ramadan, M.E. (2015). The use of medical and drug information software programs for personal digital assistants among pharmacy students in a Malaysian pharmacy school. Currents in Pharmacy Teaching and Learning, 7:484-491. https://doi.org/10.1016/j.cpt1.2015.04.015

[20] Charalmpos, D., Ilias, M., Panagiotis, T., Flora, M., and George, V. (2010). mPharmacy: A System enabling prescription and personal assistive medication management on mobile devices. Wireless Mobile Communication and Healthcare, 55:153-159

[21] Elsayed, T. M., Jamshed, S.Q., and Mohamad Elkalmi, R. (2015). The use of medical and drug information software programs for personal digital assistants among pharmacy students in a Malaysian pharmacy school, 7(4): 484-491

[22] Richard, C.A.H., and Bryant, J.E. (2014). Pharmacy student perceptions on the introduction of clinical case studies solved with Apple mobile device into a basic health science laboratory. Current in Pharmacy Teaching and Learning, 6(2014): 659-666 https://doi.org/10.1016/j.cptl.2014.05.006

\section{$7 \quad$ Acknowledgment}

This research was under the collaboration of the Embedded Systems and Mobile Application Laboratory and the Faculty of Pharmacy, Chiang Mai University. We 
would like to acknowledge the College of Arts, Media and Technology, Chiang Mai University for their financial support of the project.

\section{Authors}

Orawit Thinnukool received his Ph.D. degree in Research methodology from the Prince of Songkla University. His blackground is in information technology, education technology and research operation. Currently, he is a lecturer and a member of the Embedded Systems and Mobile Application Laboratory and the Department of Modern Management and Information Technology at the College of Art Media and Technology.

Pattaraporn Khuwuthyakorn received her Ph.D. in Engineering from the Australian National University in 2012. Her area is in information technology and engineering. Currently, she is a lecturer at the College of Arts, Media and Technology, Chiang Mai University.

Purida Wientong received her Ph.D. in Pharmaceutical Care. Her area is in healthcare system and healthcare management. Currently she is a researcher and a lecturer of the Department of Pharmaceutical Care, Faculty of Pharmacy, Chiang Mai University.

Article submitted 10 February 2017. Published as resubmitted by the authors 18 March 2017 